\title{
Quasi-static transient thermal stresses in a thick annular disc
}

\author{
V S KULKARNI ${ }^{1}$ and K C DESHMUKH ${ }^{2}$ \\ ${ }^{1}$ Department of Mathematics, Govt. College of Engineering, Chandrapur 442401 \\ ${ }^{2}$ Post-Graduate Department of Mathematics, Nagpur University, Nagpur 440010 \\ e-mail: vinayakskulkarni1@rediffmail.com; kcdeshmukh2000@rediffmail.com
}

MS received 3 August 2006; revised 31 June 2007

\begin{abstract}
The present paper deals with the determination of transient thermal stresses in a thick annular disc. A thick annular disc is considered having zero initial temperature and subjected to arbitrary heat flux on the upper and lower surfaces where as the fixed circular edges are at zero temperature.The governing heat conduction equation have been solved by using integral transform technique. The results are obtained in series form in terms of Bessel's functions. The results for displacement and stresses have been computed numerically and are illustrated graphically.
\end{abstract}

Keywords. Annular disc; thermo-elastic problem; axisymmetric; thermal stresses.

\section{Introduction}

During the second half of the twentieth century, non-isothermal problems of the theory of elasticity became increasingly important. This is due to their wide application in diverse fields. The high velocities of modern aircraft give rise to aerodynamic heating, which produces intense thermal stresses that reduce the strength of the aircraft structure.

Nowacki (1957) has determined steady-state thermal stresses in circular plate subjected to an axisymmetric temperature distribution on the upper face with zero temperature on the lower face and the circular edge. The direct thermo-elastic problem in an annular fin is studied by Shang-Sheng Wu (1997). Wankhede (1982) determined Quasi static thermal stresses in thin circular plate. Gogulwar \& Deshmukh (2002) solved the inverse problem of thermal stresses in a thin annular disc. Also Deshmukh 2002 studied transient heat conduction problem in a thin hollow cylinder and determined thermal stresses. Ching-Huang Chiu et al (2002) determined thermal stresses in annular fins with temperature-dependent conductivity under periodic heat transfer boundary condition is analysed by the Adomians decomposition method. Moreever, Qian \& Batra (2004) studied transient thermo-elastic deformation of thick functionally graded plate.

Recently Naotake Noda et al (2003) considered infinite thick circular plate and discussed thermal stresses due to arbitrary heat flux on the upper and lower Surfaces, while we consider finite thick annular disc and discussed thermal stresses. Due to arbitrary heat flux on the upper and lower surfaces, disc expands in axial direction and towards the center in radial direction. 
This is new and novel contribution of this study. No one has done such type of work on the thick annular disc so far. The results presented here are useful in engineering problem particularly in the determination of the state of strain in thick circular plate constituting foundations of containers for hot gases or liquids, in the foundations for furnaces, etc.

\section{Formulation of the problem}

Consider a thick a annular disc of thickness $2 h$ occupying space $D$ defined by $a \leq r \leq b$, $-h \leq z \leq h$. Let the disc be subjected to a transient axisymmetric temperature field on the radial and axial directions of the cylindrical coordinate system. Initially, the plate is kept at zero temperature. The arbitrary heat flux $\frac{Q f(r)}{\lambda}$ is prescribed over the upper surface $(z=h)$ and the lower surface $(z=-h)$. The fixed circular edges $(r=a$ and $r=b)$ are at zero temperature. Assume the upper and lower surface of thick annular disc are traction-free. Under these realistic prescribed conditions, the quasi-static transient thermal stresses are required to be determined.

The differential equation governing the displacement potential function $\phi(r, z, t)$ is given in Naotake Noda et al (2003) as

$$
\frac{\partial^{2} \phi}{\partial r^{2}}+\frac{1}{r} \frac{\partial \phi}{\partial r}+\frac{\partial^{2} \phi}{\partial z^{2}}=K \tau
$$

where $K$ is the restraint coefficient and temperature change $\tau=T-T_{i} . T_{i}$ is initial temperature. Displacement function $\phi$ is known as Goodier's thermo-elastic potential.

The temperature of the disc at time $t$ satisfies the heat conduction equation,

$$
\frac{\partial^{2} T}{\partial r^{2}}+\frac{1}{r} \frac{\partial T}{\partial r}+\frac{\partial^{2} T}{\partial z^{2}}=\frac{1}{k} \frac{\partial T}{\partial t}
$$

with the boundary conditions

$$
\begin{aligned}
\frac{\partial T}{\partial z} & = \pm \frac{Q f(r)}{\lambda} \text { for } z= \pm h, \quad a \leq r \leq b, \\
T & =0 \text { at } r=a,-h \leq z \leq h, \\
T & =0 \text { at } r=b,-h \leq z \leq h,
\end{aligned}
$$

and the initial condition

$$
T=0 \text { at } t=0 .
$$

where $k$ is the thermal diffusivity of the material of the disc.

The displacement function in the cylindrical coordinate system are represented by the Michell's function defined in Naotake Noda et al (2003) as

$$
u_{r}=\frac{\partial \phi}{\partial r}-\frac{\partial^{2} M}{\partial r \partial z}
$$

and

$$
u_{z}=\frac{\partial \phi}{\partial z}+2(1-v) \nabla^{2} M-\frac{\partial^{2} M}{\partial z^{2}} .
$$


The Michell's function must satisfy

$$
\nabla^{2} \nabla^{2} M=0
$$

where

$$
\nabla^{2}=\frac{\partial^{2}}{\partial r^{2}}+\frac{1}{r} \cdot \frac{\partial}{\partial r}+\frac{\partial^{2}}{\partial z^{2}} .
$$

The component of the stresses are represented by the thermo-elastic displacement potential $\phi$ and Michell's function $M$ as

$$
\begin{aligned}
& \sigma_{r r}=2 G\left[\frac{\partial^{2} \phi}{\partial r^{2}}-K \tau+\frac{\partial}{\partial z}\left(v \nabla^{2} M-\frac{\partial^{2} M}{\partial r^{2}}\right)\right], \\
& \sigma_{\theta \theta}=2 G\left[\frac{1}{r} \frac{\partial \phi}{\partial r}-K \tau+\frac{\partial}{\partial z}\left(v \nabla^{2} M-\frac{1}{r} \frac{\partial M}{\partial r}\right)\right], \\
& \sigma_{z z}=2 G\left[\frac{\partial^{2} \phi}{\partial z^{2}}-K \tau+\frac{\partial}{\partial z}\left((2-v) \nabla^{2} M-\frac{\partial^{2} M}{\partial z^{2}}\right)\right],
\end{aligned}
$$

and

$$
\sigma_{r z}=2 G\left[\frac{\partial^{2} \phi}{\partial r \partial z}+\frac{\partial}{\partial r}\left((1-v) \nabla^{2} M-\frac{\partial^{2} M}{\partial z^{2}}\right)\right],
$$

where $G$ and $v$ are the shear modulus and Poisson's ratio respectively.

For traction-free surfaces the stress functions

$$
\sigma_{z z}=\sigma_{r z}=0 \text { at } z= \pm h .
$$

Equations (1) to (15) constitute mathematical formulation of the problem.

\section{Solution}

Taking Laplace transform of equations (2) to (5) with respect to $t$ and using equation (6), one obtains

$$
\frac{\partial^{2} \bar{T}}{\partial r^{2}}+\frac{1}{r} \frac{\partial \bar{T}}{\partial r}+\frac{\partial^{2} \bar{T}}{\partial z^{2}}=\frac{P}{k} \bar{T}
$$

with boundary conditions

$$
\begin{gathered}
\frac{\partial \bar{T}}{\partial z}= \pm \frac{Q f(r)}{\lambda P} \text { at } z= \pm h, \\
\bar{T}=0 \text { at } r=a \& r=b
\end{gathered}
$$

where $P$ is Laplace transform parameter $\& \bar{T}$ is Laplace transform of $T$. 
Introduce the finite Hankal transform over the variable $r$ and its inverse transform defined as in (Sneddon 1972)

$$
\begin{gathered}
\overline{\bar{T}}\left(\alpha_{m}, z\right)=\int_{a}^{b} r K_{0}\left(\alpha_{m}, r\right) \bar{T}(r, z) d r \\
\bar{T}(r, z)=\sum_{m=1}^{\infty} \overline{\bar{T}}\left(\alpha_{m}, z\right) K_{0}\left(\alpha_{m}, r\right)
\end{gathered}
$$

where

$$
\begin{aligned}
& K_{0}\left(\alpha_{m}, r\right)=\frac{R_{0}\left(\alpha_{m}, r\right)}{\sqrt{N}}, \\
& R_{0}\left(\alpha_{m}, r\right)=\frac{J_{0}\left(\alpha_{m} r\right)}{J_{0}\left(\alpha_{m} b\right)}-\frac{Y_{0}\left(\alpha_{m} r\right)}{Y_{0}\left(\alpha_{m} b\right)},
\end{aligned}
$$

The normality constant

$$
N=\frac{b^{2}}{2} \dot{R}_{0}^{2}\left(\alpha_{m}, b\right)-\frac{a^{2}}{2} \dot{\bullet}_{0}^{2}\left(\alpha_{m}, a\right)
$$

and $\alpha_{1}, \alpha_{2} \ldots$ are roots of the transcendental equation

$$
\frac{J_{0}(\alpha a)}{J_{0}(\alpha b)}-\frac{Y_{0}(\alpha a)}{Y_{0}(\alpha b)}=0
$$

$J_{n}(x)$ is Bessel function of the first kind of order $n \& Y_{n}(x)$ is Bessel function of the second kind of order $n$.

This transform satisfies the relation

$$
H\left[\frac{\partial^{2} \bar{T}}{\partial r^{2}}+\frac{1}{r} \frac{\partial \bar{T}}{\partial r}\right]=-\alpha_{m}^{2} \overline{\bar{T}}\left(\alpha_{m}, z\right)
$$

and

$$
H\left[\frac{\partial^{2} \bar{T}}{\partial z^{2}}\right]=\frac{d^{2} \overline{\bar{T}}}{d z^{2}}
$$

On applying the finite Hankal transform defined in the equation (19) to the equation (16), one obtains

$$
\frac{d^{2} \overline{\bar{T}}}{d z^{2}}-\alpha_{m}^{2} \overline{\bar{T}}=\frac{P}{k} \overline{\bar{T}}
$$

where $\overline{\bar{T}}$ is the Hankal transform of $\bar{T}$.

On solving equation (27) under condition given in equation (17), one obtains

$$
\overline{\bar{T}}=\sum_{m=1}^{\infty} A_{m} \cosh \left[\left(\sqrt{\alpha_{m}^{2}+q^{2}}\right) Z\right]
$$

where $q^{2}=\frac{P}{K}$. 
Now constant $A_{m}$ can be obtained by using equations (17) and (28)

$$
A_{m}=\frac{Q \bar{f}\left(\alpha_{m}\right)}{\lambda P \sqrt{\alpha_{m}^{2}+q^{2}} \sinh \left[\left(\sqrt{\alpha_{m}^{2}+q^{2}}\right) h\right]}
$$

Thus equation (28) becomes

$$
\overline{\bar{T}}=\frac{Q}{\lambda} \sum_{m=1}^{\infty}\left[\frac{\bar{f}\left(\alpha_{m}\right) \cosh \left[\left(\sqrt{\alpha_{m}^{2}+q^{2}}\right) Z\right]}{P \sqrt{\alpha_{m}^{2}+q^{2}} \sinh \left[\left(\sqrt{\alpha_{m}^{2}+q^{2}}\right) h\right]}\right]
$$

On applying inverse Hankal transform defined in equation (20), one obtains

$$
\bar{T}=\left(\frac{Q}{\lambda}\right) \sum_{n=1}^{\infty}\left(\frac{\bar{f}\left(\alpha_{m}\right)}{\sqrt{N}}\right)\left[\frac{J_{0}\left(\alpha_{m} r\right)}{J_{0}\left(\alpha_{m} b\right)}-\frac{Y_{0}\left(\alpha_{m} r\right)}{Y_{0}\left(\alpha_{m} b\right)}\right]\left[\frac{\cosh \left[\left(\sqrt{\alpha_{m}^{2}+q^{2}}\right) Z\right]}{P \sqrt{\alpha_{m}^{2}+q^{2}} \sinh \left[\left(\sqrt{\alpha_{m}^{2}+q^{2}}\right) h\right]}\right]
$$

where $\bar{f}\left(\alpha_{m}\right)$ is Hankal transform of $f(r)$.

Finally, applying inverse Laplace transform on equation (31) one obtains the expression for temperature as,

$$
\begin{aligned}
T= & \left(\frac{Q}{\lambda}\right) \sum_{m=1}^{\infty}\left(\frac{\bar{f}\left(\alpha_{m}\right)}{\sqrt{N}}\right)\left[\frac{J_{0}\left(\alpha_{m} r\right)}{J_{0}\left(\alpha_{m} b\right)}-\frac{Y_{0}\left(\alpha_{m} r\right)}{Y_{0}\left(\alpha_{m} b\right)}\right] \\
& \times\left[\frac{\cosh \left(\alpha_{m} z\right)}{\alpha_{m} \sinh \left(\alpha_{m} h\right)}-\frac{e^{-\alpha_{m}^{2} k t}}{\alpha_{m}^{2} h}+2 h \sum_{n=1}^{\infty} \frac{\left.(-1)^{n+1} \cos \left(\frac{n \pi z}{h}\right) e^{-k\left[\left(n^{2} \pi^{2}+\alpha_{m}^{2} h^{2}\right) \frac{t}{h^{2}}\right.}\right]}{\left(n^{2} \pi^{2}+\alpha_{m}^{2} h^{2}\right)}\right]
\end{aligned}
$$

Since initial temperature $T_{i}=0, \tau=T$

Now assume Michell's function $M$ which satisfies condition (9) as,

$$
\begin{aligned}
M= & \left(\frac{Q K}{\lambda}\right) \sum_{m=1}^{\infty} \sum_{n=1}^{\infty}\left\{\left(\frac{\bar{f}\left(\alpha_{m}\right)}{\sqrt{N}}\right)\left[\frac{J_{0}\left(\alpha_{m} r\right)}{J_{0}\left(\alpha_{m} b\right)}-\frac{Y_{0}\left(\alpha_{m} r\right)}{Y_{0}\left(\alpha_{m} b\right)}\right]\right. \\
& \left.\times\left[B_{m n} \sinh \left(\alpha_{m} z\right)+C_{m n} \alpha_{m} z \cosh \left(\alpha_{m} z\right)\right]\right\}
\end{aligned}
$$

where $B_{m n}$ and $C_{m n}$ are the arbitrary functions, which can be determined finally by using condition (15). 
To obtain displacement potential $\phi$ using equations (32) and (33) in equation (1) one have,

$$
\begin{aligned}
\frac{\partial^{2} \phi}{\partial r^{2}}+ & \frac{1}{r} \frac{\partial \phi}{\partial r}+\frac{\partial^{2} \phi}{\partial z^{2}} \\
= & \left(\frac{Q K}{\lambda}\right) \sum_{m=1}^{\infty}\left(\frac{\bar{f}\left(\alpha_{m}\right)}{\sqrt{N}}\right)\left[\frac{J_{0}\left(\alpha_{m} r\right)}{J_{0}\left(\alpha_{m} b\right)}-\frac{Y_{0}\left(\alpha_{m} r\right)}{Y_{0}\left(\alpha_{m} b\right)}\right] \\
& \times\left[\frac{\cosh \left(\alpha_{m} z\right)}{\alpha_{m} \sinh \left(\alpha_{m} h\right)}-\frac{e^{-\alpha_{m}^{2} k t}}{\alpha_{m}^{2} h}+2 h \sum_{n=1}^{\infty} \frac{(-1)^{n+1} \cos \left(\frac{n \pi z}{h}\right) e^{-k\left[\left(n^{2} \pi^{2}+\alpha_{m}^{2} h^{2}\right) \frac{t}{h^{2}}\right]}}{\left(n^{2} \pi^{2}+\alpha_{m}^{2} h^{2}\right)}\right]
\end{aligned}
$$

Considering first term of equation (35) as

$$
\frac{\partial^{2} \phi_{1}}{\partial r^{2}}+\frac{1}{r} \frac{\partial \phi_{1}}{\partial r}+\frac{\partial^{2} \phi_{1}}{\partial z^{2}}=\left(\frac{Q K}{\lambda}\right) \sum_{m=1}^{\infty}\left(\frac{\bar{f}\left(\alpha_{m}\right)}{\sqrt{N}}\right)\left[\frac{J_{0}\left(\alpha_{m} r\right)}{J_{0}\left(\alpha_{m} b\right)}-\frac{Y_{0}\left(\alpha_{m} r\right)}{Y_{0}\left(\alpha_{m} b\right)}\right]\left[\frac{\cosh \left(\alpha_{m} z\right)}{\alpha_{m} \sinh \left(\alpha_{m} h\right)}\right]
$$

To solve equation (36) assume

$$
\phi_{1}=\sum_{m=1}^{\infty}\left\{D_{m} \frac{\bar{f}\left(\alpha_{m}\right)}{\sqrt{N}}\left[\frac{J_{0}\left(\alpha_{m} r\right)}{J_{0}\left(\alpha_{m} b\right)}-\frac{Y_{0}\left(\alpha_{m} r\right)}{Y_{0}\left(\alpha_{m} b\right)}\right]\left[z \sinh \left(\alpha_{m} z\right)\right]\right\}
$$

Using equation (37) in equation (36) one obtains

$$
D_{m}=\frac{Q K \bar{f}\left(\alpha_{m}\right)}{2 \lambda \sqrt{N} \alpha_{m}^{2} \sinh \left(\alpha_{m} h\right)}
$$

Hence

$$
\phi_{1}=\left(\frac{Q K}{2 \lambda}\right) \sum_{m=1}^{\infty}\left(\frac{\bar{f}\left(\alpha_{m}\right)}{\alpha_{m}^{2} \sqrt{N}}\right)\left[\frac{J_{0}\left(\alpha_{m} r\right)}{J_{0}\left(\alpha_{m} b\right)}-\frac{Y_{0}\left(\alpha_{m} r\right)}{Y_{0}\left(\alpha_{m} b\right)}\right]\left[\frac{z \sinh \left(\alpha_{m} z\right)}{\sinh \left(\alpha_{m} h\right)}\right]
$$

Now considering second and third term of equation (35) as

$$
\begin{aligned}
\frac{\partial^{2} \phi_{2}}{\partial r^{2}} & +\frac{1}{r} \frac{\partial \phi_{2}}{\partial r}+\frac{\partial^{2} \phi_{2}}{\partial z^{2}} \\
= & \left(\frac{Q K}{\lambda}\right) \sum_{m=1}^{\infty}\left(\frac{\bar{f}\left(\alpha_{m}\right)}{\sqrt{N}}\right)\left[\frac{J_{0}\left(\alpha_{m} r\right)}{J_{0}\left(\alpha_{m} b\right)}-\frac{Y_{0}\left(\alpha_{m} r\right)}{Y_{0}\left(\alpha_{m} b\right)}\right] \\
& \times\left[\frac{-e^{-\alpha_{m}^{2} k t}}{\alpha_{m}^{2} h}+2 h \sum_{n=1}^{\infty} \frac{(-1)^{n+1} \cos \left(\frac{n \pi z}{h}\right) e^{-k\left[\left(n^{2} \pi^{2}+\alpha_{m}^{2} h^{2}\right) \frac{t}{h^{2}}\right]}}{\left(n^{2} \pi^{2}+\alpha_{m}^{2} h^{2}\right)}\right]
\end{aligned}
$$

To solve equation (39) using

$$
\frac{\partial^{2}}{\partial r^{2}}+\frac{1}{r} \frac{\partial}{\partial r}+\frac{\partial^{2}}{\partial z^{2}} \approx \frac{1}{k} \frac{\partial}{\partial t} \text { in equation (39) }
$$


and on integrating with respect to $t$, one obtains

$$
\begin{aligned}
\phi_{2}= & \left(\frac{Q K}{\lambda}\right) \sum_{m=1}^{\infty}\left(\frac{\bar{f}\left(\alpha_{m}\right)}{\sqrt{N}}\right)\left[\frac{J_{0}\left(\alpha_{m} r\right)}{J_{0}\left(\alpha_{m} b\right)}-\frac{Y_{0}\left(\alpha_{m} r\right)}{Y_{0}\left(\alpha_{m} b\right)}\right] \\
& \times\left[\frac{e^{-\alpha_{m}^{2} k t}}{\alpha_{m}^{4} h}+2 h^{3} \sum_{n=1}^{\infty} \frac{(-1)^{n} \cos \left(\frac{n \pi z}{h}\right) e^{-k\left[\left(n^{2} \pi^{2}+\alpha_{m}^{2} h^{2}\right) \frac{t}{h^{2}}\right]}}{\left(n^{2} \pi^{2}+\alpha_{m}^{2} h^{2}\right)^{2}}\right]
\end{aligned}
$$

Finally $\phi=\phi_{1}+\phi_{2}$

$$
\begin{aligned}
\phi= & \left(\frac{Q K}{\lambda}\right) \sum_{m=1}^{\infty}\left(\frac{\bar{f}\left(\alpha_{m}\right)}{\sqrt{N}}\right)\left[\frac{J_{0}\left(\alpha_{m} r\right)}{J_{0}\left(\alpha_{m} b\right)}-\frac{Y_{0}\left(\alpha_{m} r\right)}{Y_{0}\left(\alpha_{m} b\right)}\right] \\
& \times\left\{\frac{z \sinh \left(\alpha_{m} z\right)}{2 \alpha_{m}^{2} \sinh \left(\alpha_{m} h\right)}+\frac{e^{-\alpha_{m}^{2} k t}}{\alpha_{m}^{4} h} 2 h^{3} \sum_{n=1}^{\infty} \frac{(-1)^{n} \cos \left(\frac{n \pi z}{h}\right) e^{-k\left[\left(n^{2} \pi^{2}+\alpha_{m}^{2} h^{2}\right) \frac{t}{h^{2}}\right]}}{\left(n^{2} \pi^{2}+\alpha_{m}^{2} h^{2}\right)^{2}}\right\}
\end{aligned}
$$

Now using equations (32), (33), (34) and (42) in equations (7), (8) and (11) to (14), One obtains the expressions for displacement and stresses as

$$
\begin{aligned}
u_{r}= & \left(\frac{Q K}{\lambda}\right) \sum_{m=1}^{\infty}\left(\frac{\bar{f}\left(\alpha_{m}\right)}{\sqrt{N}}\right)\left[\frac{J_{1}\left(\alpha_{m} r\right)}{J_{0}\left(\alpha_{m} b\right)}-\frac{Y_{1}\left(\alpha_{m} r\right)}{Y_{0}\left(\alpha_{m} b\right)}\right]\left\{\frac{-z \sinh \left(\alpha_{m} z\right)}{2 \alpha_{m} \sinh \left(\alpha_{m} h\right)}\right. \\
& -\frac{e^{-\alpha_{m}^{2} k t}}{\alpha_{m}^{3} h}-2 \alpha_{m} h^{3} \sum_{n=1}^{\infty} \frac{(-1)^{n} \cos \left(\frac{n \pi z}{h}\right) e^{-k\left[\left(n^{2} \pi^{2}+\alpha_{m}^{2} h^{2}\right) \frac{t}{h^{2}}\right]}}{\left(n^{2} \pi^{2}+\alpha_{m}^{2} h^{2}\right)^{2}} \\
& \left.+\alpha_{m}^{2} B_{m n} \cosh \left(\alpha_{m} z\right)+\alpha_{m}^{2} C_{m n}\left[\cosh \left(\alpha_{m} z\right)+\alpha_{m} z \sinh \left(\alpha_{m} z\right)\right]\right\} \\
u_{z}= & \left(\frac{Q K}{\lambda}\right) \sum_{m=1}^{\infty}\left(\frac{\bar{f}\left(\alpha_{m}\right)}{\sqrt{N}}\right)\left[\frac{J_{0}\left(\alpha_{m} r\right)}{J_{0}\left(\alpha_{m} b\right)}-\frac{Y_{0}\left(\alpha_{m} r\right)}{Y_{0}\left(\alpha_{m} b\right)}\right]\left\{\frac{\sinh \left(\alpha_{m} z\right)+\alpha_{m} z \cosh \left(\alpha_{m} z\right)}{2 \alpha_{m}^{2} \sinh \left(\alpha_{m} h\right)}\right. \\
& -2 h^{2} \pi \sum_{n=1}^{\infty} \frac{(-1)^{n} n \sin \left(\frac{n \pi z}{h}\right) e^{-k\left[\left(n^{2} \pi^{2}+\alpha_{m}^{2} h^{2}\right) \frac{t}{h^{2}}\right]}}{\left(n^{2} \pi^{2}+\alpha_{m}^{2} h^{2}\right)^{2}} \\
& \left.-\alpha_{m}^{2} B_{m n} \sinh \left(\alpha_{m} z\right)+\alpha_{m}^{2} C_{m n}\left[2(1-2 v) \sinh \left(\alpha_{m} z\right)-\alpha_{m} z \cosh \left(\alpha_{m} z\right)\right]\right\}
\end{aligned}
$$




$$
\begin{aligned}
& \sigma_{r r}=\left(\frac{2 G Q K}{\lambda}\right) \sum_{m=1}^{\infty}\left(\frac{\bar{f}\left(\alpha_{m}\right)}{\sqrt{N}}\right)\left\{[ \frac { \dot { J } _ { 1 } ( \alpha _ { m } r ) } { J _ { 0 } ( \alpha _ { m } b ) } - \frac { \dot { Y } _ { 1 } ( \alpha _ { m } r ) } { Y _ { 0 } ( \alpha _ { m } b ) } ] \left[\frac{-z \sinh \left(\alpha_{m} z\right)}{2 \alpha_{m} \sinh \left(\alpha_{m} h\right)}\right.\right. \\
& \left.-\frac{e^{-\alpha_{m}^{2} k t}}{\alpha_{m}^{3} h}-2 \alpha_{m} h^{3} \sum_{n=1}^{\infty} \frac{(-1)^{n} \cos \left(\frac{n \pi z}{h}\right) e^{-k\left[\left(n^{2} \pi^{2}+\alpha_{m}^{2} h^{2}\right) \frac{t}{h^{2}}\right]}}{\left(n^{2} \pi^{2}+\alpha_{m}^{2} h^{2}\right)^{2}}\right] \\
& -\left[\frac{J_{0}\left(\alpha_{m} r\right)}{J_{0}\left(\alpha_{m} b\right)}-\frac{Y_{0}\left(\alpha_{m} r\right)}{Y_{0}\left(\alpha_{m} b\right)}\right]\left[\frac{\cosh \left(\alpha_{m} z\right)}{\alpha_{m} \sinh \left(\alpha_{m} h\right)}-\frac{e^{-\alpha_{m}^{2} k t}}{\alpha_{m}^{2} h}\right. \\
& \left.+2 h \sum_{n=1}^{\infty} \frac{(-1)^{n+1} \cos \left(\frac{n \pi z}{h}\right) e^{-k\left[\left(n^{2} \pi^{2}+\alpha_{m}^{2} h^{2}\right) \frac{t}{h^{2}}\right]}}{\left(n^{2} \pi^{2}+\alpha_{m}^{2} h^{2}\right)}\right] \\
& +B_{m n} \alpha_{m}^{2}\left[\frac{\dot{J}_{1}\left(\alpha_{m} r\right)}{J_{0}\left(\alpha_{m} b\right)}-\frac{\dot{Y}_{1}\left(\alpha_{m} r\right)}{Y_{0}\left(\alpha_{m} b\right)}\right] \cosh \left(\alpha_{m} z\right) \\
& \left.+C_{m n} \alpha_{m}^{2}\left[\begin{array}{l}
2 v \alpha_{m}\left[\frac{J_{0}\left(\alpha_{m} r\right)}{J_{0}\left(\alpha_{m} b\right)}-\frac{Y_{0}\left(\alpha_{m} r\right)}{Y_{0}\left(\alpha_{m} b\right)}\right] \cosh \left(\alpha_{m} z\right) \\
+\left[\frac{\dot{J}_{1}\left(\alpha_{m} r\right)}{J_{0}\left(\alpha_{m} b\right)}-\frac{\dot{Y}\left(\alpha_{m} r\right)}{Y_{0}\left(\alpha_{m} b\right)}\right]\left[\cosh \left(\alpha_{m} z\right)+\left(\alpha_{m} z\right) \sinh \left(\alpha_{m} z\right)\right.
\end{array}\right]\right\} \\
& \left.\sigma_{\theta \theta}=\left(\frac{2 G Q K}{\lambda}\right) \sum_{m=1}^{\infty}\left(\frac{\bar{f}\left(\alpha_{m}\right)}{\sqrt{N}}\right)\right]\left\{\frac{1}{r}\left[\frac{J_{1}\left(\alpha_{m} r\right)}{J_{0}\left(\alpha_{m} b\right)}-\frac{Y_{1}\left(\alpha_{m} r\right)}{Y_{0}\left(\alpha_{m} b\right)}\right]\right. \\
& \times\left[\frac{-z \sinh \left(\alpha_{m} z\right)}{2 \alpha_{m} \sinh \left(\alpha_{m} h\right)}-\frac{e^{-\alpha_{m}^{2} k t}}{\alpha_{m}^{3} h}\right. \\
& \left.-2 \alpha_{m} h^{3} \sum_{n=1}^{\infty} \frac{(-1)^{n} \cos \left(\frac{n \pi z}{h}\right) e^{-k\left[\left(n^{2} \pi^{2}+\alpha_{m}^{2} h^{2}\right) \frac{t}{h^{2}}\right]}}{\left(n^{2} \pi^{2}+\alpha_{m}^{2} h^{2}\right)^{2}}\right]
\end{aligned}
$$




$$
\begin{aligned}
& -\left[\frac{J_{0}\left(\alpha_{m} r\right)}{J_{0}\left(\alpha_{m} b\right)}-\frac{Y_{0}\left(\alpha_{m} r\right)}{Y_{0}\left(\alpha_{m} b\right)}\right]\left[\frac{\cosh \left(\alpha_{m} z\right)}{\alpha_{m} \sinh \left(\alpha_{m} h\right)}-\frac{e^{-\alpha_{m}^{2} k t}}{\alpha_{m}^{2} h}\right. \\
& \left.+2 h \sum_{n=1}^{\infty} \frac{(-1)^{n+1} \cos \left(\frac{n \pi z}{h}\right) e^{-k\left[\left(n^{2} \pi^{2}+\alpha_{m}^{2} h^{2}\right) \frac{t}{h^{2}}\right]}}{\left(n^{2} \pi^{2}+\alpha_{m}^{2} h^{2}\right)}\right] \\
& +\frac{B_{m n} \alpha_{m}^{2}}{r}\left[\frac{J_{1}\left(\alpha_{m} r\right)}{J_{0}\left(\alpha_{m} b\right)}-\frac{Y_{1}\left(\alpha_{m} r\right)}{Y_{0}\left(\alpha_{m} b\right)}\right] \cosh \left(\alpha_{m} z\right) \\
& +C_{m n} \alpha_{m}^{2}\left[\begin{array}{c}
2 v \alpha_{m}\left[\frac{J_{0}\left(\alpha_{m} r\right)}{J_{0}\left(\alpha_{m} b\right)}-\frac{Y_{0}\left(\alpha_{m} r\right)}{Y_{0}\left(\alpha_{m} b\right)}\right] \cosh \left(\alpha_{m} z\right) \\
+\frac{1}{r}\left[\frac{J_{1}\left(\alpha_{m} r\right)}{J_{0}\left(\alpha_{m} b\right)}-\frac{Y_{1}\left(\alpha_{m} r\right)}{Y_{0}\left(\alpha_{m} b\right)}\right]\left[\cosh \left(\alpha_{m} z\right)+\left(\alpha_{m} z\right) \sinh \left(\alpha_{m} z\right)\right.
\end{array}\right]
\end{aligned}
$$

$$
\begin{aligned}
\sigma_{z z}= & \left(\frac{2 G Q K}{\lambda}\right) \sum_{m=1}^{\infty}\left(\frac{\bar{f}\left(\alpha_{m}\right)}{\sqrt{N}}\right)\left[\frac{J_{0}\left(\alpha_{m} r\right)}{J_{0}\left(\alpha_{m} b\right)}-\frac{Y_{0}\left(\alpha_{m} r\right)}{Y_{0}\left(\alpha_{m} b\right)}\right]\left\{\frac{z \sinh \left(\alpha_{m} z\right)}{2 \sinh \left(\alpha_{m} h\right)}+\frac{e^{-\alpha_{m}^{2} k t}}{\alpha_{m}^{2} h}\right. \\
& +2 \alpha_{m}^{2} h^{3} \sum_{n=1}^{\infty} \frac{(-1)^{n} \cos \left(\frac{n \pi z}{h}\right) e^{-k\left[\left(n^{2} \pi^{2}+\alpha_{m}^{2} h^{2}\right) \frac{t}{h^{2}}\right]}}{\left(n^{2} \pi^{2}+\alpha_{m}^{2} h^{2}\right)^{2}} \\
& \left.-\alpha_{m}^{3} B_{m n} \cosh \left(\alpha_{m} z\right)+\alpha_{m}^{3} C_{m n}\left[(1-2 \nu) \cosh \left(\alpha_{m} z\right)-\alpha_{m} z \sinh \left(\alpha_{m} z\right)\right]\right\}
\end{aligned}
$$$$
\sigma_{r z}=\left(\frac{2 G Q K}{\lambda}\right) \sum_{m=1}^{\infty}\left(\frac{\bar{f}\left(\alpha_{m}\right)}{\sqrt{N}}\right)\left[\frac{J_{1}\left(\alpha_{m} r\right)}{J_{0}\left(\alpha_{m} b\right)}-\frac{Y_{1}\left(\alpha_{m} r\right)}{Y_{0}\left(\alpha_{m} b\right)}\right]
$$$$
\times\left\{\frac{-\sinh \left(\alpha_{m} z\right)-\alpha_{m} z \cosh \left(\alpha_{m} z\right)}{2 \alpha_{m} \sinh \left(\alpha_{m} h\right)}\right.
$$$$
+2 h^{2} \pi \alpha_{m} \sum_{n=1}^{\infty} \frac{(-1)^{n} n \sin \left(\frac{n \pi z}{h}\right) e^{-k\left[\left(n^{2} \pi^{2}+\alpha_{m}^{2} h^{2}\right) \frac{t}{h^{2}}\right]}}{\left(n^{2} \pi^{2}+\alpha_{m}^{2} h^{2}\right)^{2}}
$$$$
\left.+\alpha_{m}^{3} B_{m n} \sinh \left(\alpha_{m} z\right)+\alpha_{m}^{3} C_{m n}\left[2 v \sinh \left(\alpha_{m} z\right)+\left(\alpha_{m} z\right) \cosh \left(\alpha_{m} z\right)\right]\right\}
$$ 
In order to satisfy condition (15), solving equations (47) and (48) for $B_{m n}$ and $C_{m n}$ one obtains

$$
\begin{aligned}
B_{m n}= & \sum_{m=1}^{\infty}\left\{\frac{1-2 v}{2 \alpha_{m}^{4} \sinh \left(\alpha_{m} h\right)}+\frac{2 v \sinh \left(\alpha_{m} h\right)+\alpha_{m} h \cosh \left(\alpha_{m} h\right)}{\alpha_{m}^{4}\left[\sinh \left(\alpha_{m} h\right) \cosh \left(\alpha_{m} h\right)+\alpha_{m} h\right]}\right. \\
& \left.\times\left[\frac{e^{-\alpha_{m}^{2} k t}}{\alpha_{m} h}+2 \alpha_{m}^{3} h^{3} \sum_{n=1}^{\infty} \frac{e^{-k\left[\left(n^{2} \pi^{2}+\alpha_{m}^{2} h^{2}\right) \frac{t}{h^{2}}\right]}}{\left(n^{2} \pi^{2}+\alpha_{m}^{2} h^{2}\right)^{2}}\right]\right\}
\end{aligned}
$$

and

$$
\begin{aligned}
C_{m n}= & \sum_{m=1}^{\infty}\left\{\frac{1}{2 \alpha_{m}^{4} \sinh \left(\alpha_{m} h\right)}-\frac{\sinh \left(\alpha_{m} h\right)}{\alpha_{m}^{4}\left[\sinh \left(\alpha_{m} h\right) \cosh \left(\alpha_{m} h\right)+\alpha_{m} h\right]}\right. \\
& \left.\times\left[\frac{e^{-\alpha_{m}^{2} k t}}{\alpha_{m} h}+2 \alpha_{m}^{3} h^{3} \sum_{n=1}^{\infty} \frac{e^{-k\left[\left(n^{2} \pi^{2}+\alpha_{m}^{2} h^{2}\right) \frac{t}{h^{2}}\right]}}{\left(n^{2} \pi^{2}+\alpha_{m}^{2} h^{2}\right)^{2}}\right]\right\}
\end{aligned}
$$

\section{Numerical calculations}

Setting

$$
f(r)=\left(r^{2}-a^{2}\right)\left(r^{2}-b^{2}\right)
$$

Applying finite Hankal transform as defined in equations (19) to (26), one obtains

$$
\begin{aligned}
& \bar{f}\left(\alpha_{m}\right)=\int_{a}^{b} \frac{1}{\sqrt{N}}\left[\frac{J_{0}\left(\alpha_{m} r\right)}{J_{0}\left(\alpha_{m} b\right)}-\frac{Y_{0}\left(\alpha_{m} r\right)}{Y_{0}\left(\alpha_{m} b\right)}\right] r\left(r^{2}-a^{2}\right)\left(r^{2}-b^{2}\right) d r \\
& \bar{f}\left(\alpha_{m}\right)=\frac{8\left\{\left(a^{2} \alpha_{m}^{2}-3 b^{2} \alpha_{m}^{2}+16\right) J_{0}\left(\alpha_{m} a\right)-\left(b^{2} \alpha_{m}^{2}-3 a^{2} \alpha_{m}^{2}+16\right) J_{0}\left(\alpha_{m} b\right)\right\}}{\pi \sqrt{N} \alpha_{m}^{6} J_{0}\left(\alpha_{m} a\right) J_{0}\left(\alpha_{m} b\right) Y_{0}\left(\alpha_{m} b\right)}
\end{aligned}
$$

The numerical calculations have been carried out for steel $\left(\mathrm{SN} 50^{\circ} \mathrm{C}\right)$ plate with the parameters $a=1 \mathrm{~m}, b=2 \mathrm{~m}, h=0.3 \mathrm{~m}$. thermal diffusivity $k=15.9 \times 10^{-6}\left(\mathrm{~m}^{2} \mathrm{~s}^{-1}\right)$ and poisson ratio $v=0.281$ with $\alpha_{1}=3 \cdot 120, \alpha_{2}=6.2734, \alpha_{3}=9.4182, \alpha_{4}=12.5614, \alpha_{5}=15.7040$ are the roots of transdental equation $\left[\frac{J_{0}(\alpha a)}{J_{0}(\alpha b)}-\frac{Y_{0}(\alpha a)}{Y_{0}(\alpha b)}\right]=0$.

For convenience setting $A=\frac{Q K}{10^{5} \pi \lambda}, B=\frac{2 G Q K}{10^{5} \pi \lambda}$. in the expressions (43) to (48). The numerical expressions for temperature, displacement and stress components are obtained by equations (32) \& (43) to (48).

In order to examine the influence of heat flux on the upper and lower surface of thick plate, one performed the numerical calculations $r=1,1.2,1.4,1.6,1.8$ and 2, $z=-0.3,-0 \cdot 15,0,0.15$ and 0.3 and $t=5$. Numerical variations in radial and axial directions are shown in the figures with the help of computer programme (figures 1-12). 


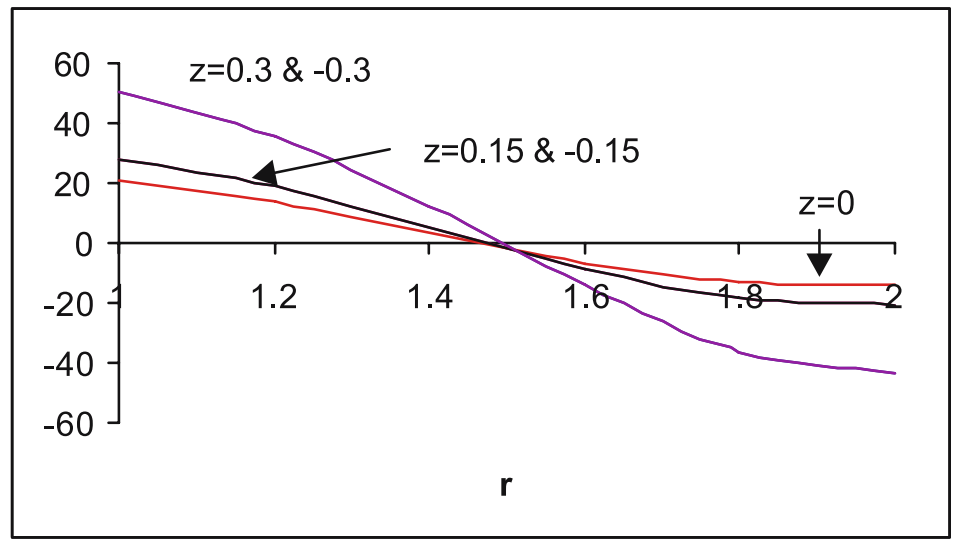

Figure 1. The radial displacement function $u_{r} / A$ in radial direction.

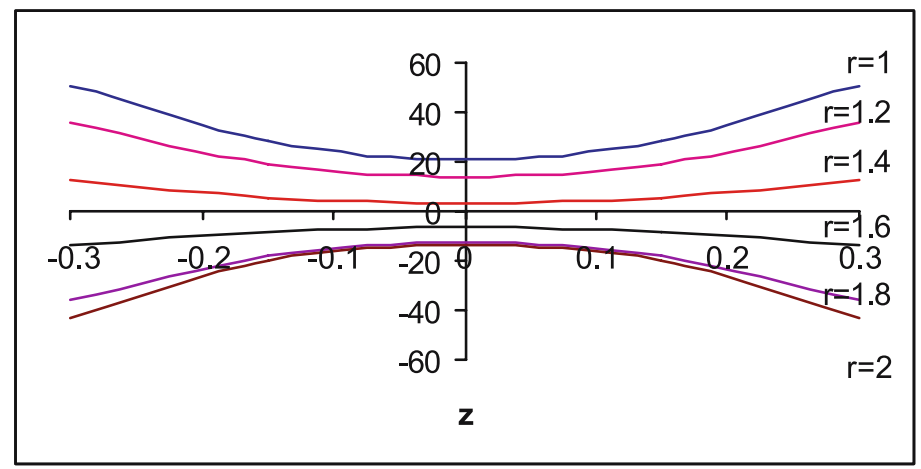

Figure 2. The radial displacement function $u_{r} / A$ in axial direction.

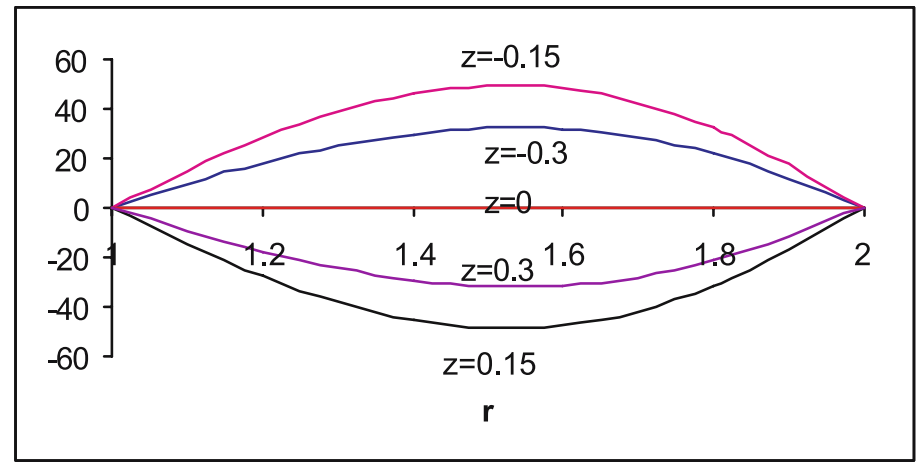

Figure 3. The axial displacement function $u_{z} / A$ in radial axial direction. 


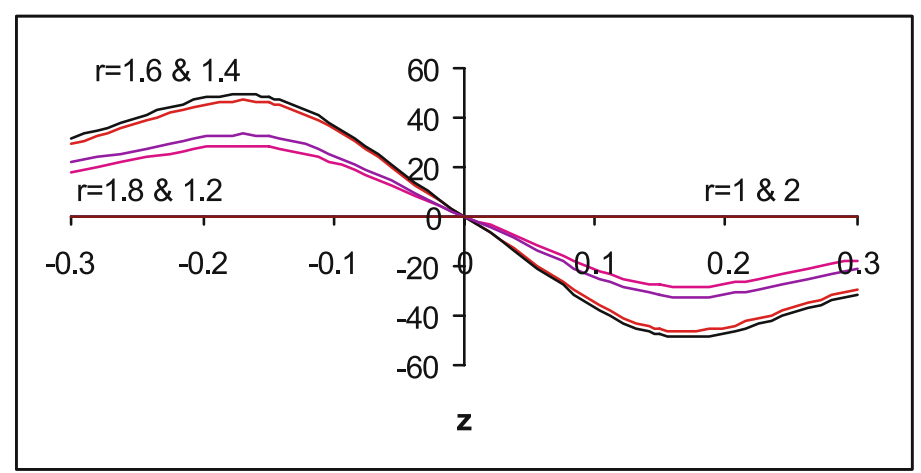

Figure 4. The axial displacement function $u_{z} / A$ in axial direction.

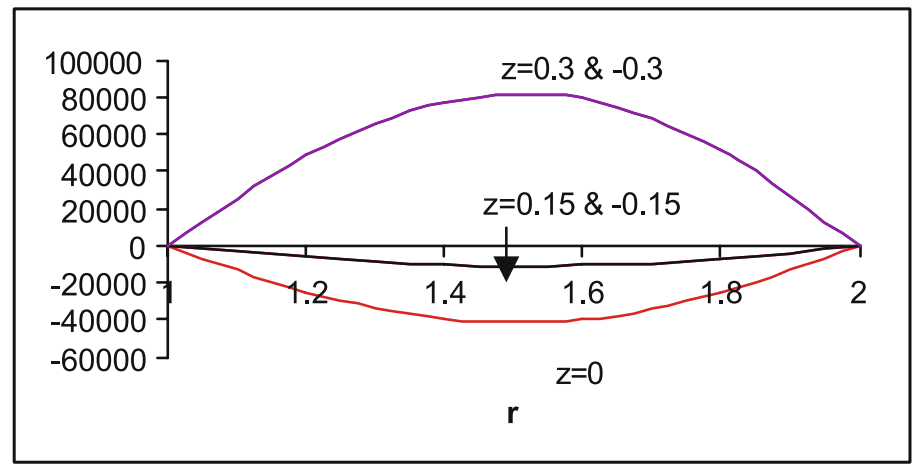

Figure 5. The radial stress function $\sigma_{r r} / B$ in radial direction.

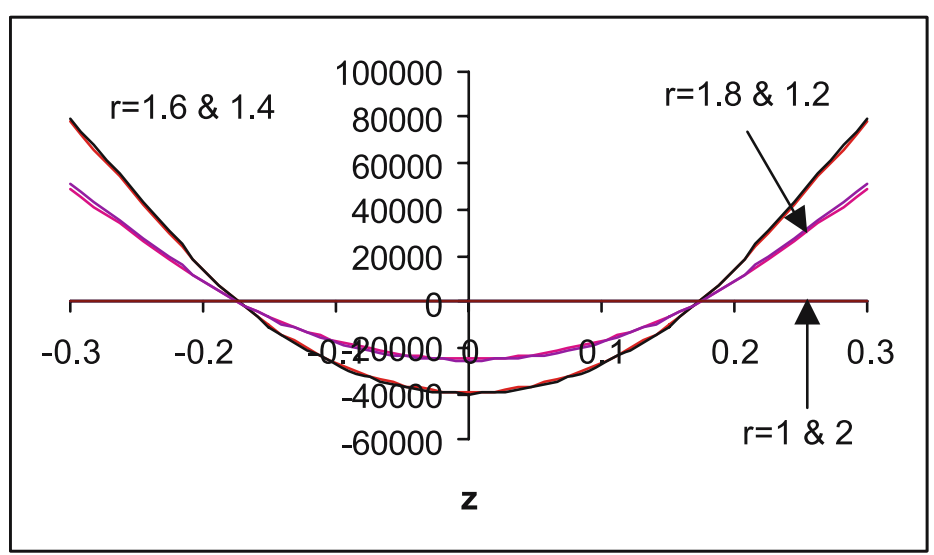

Figure 6. The radial stress function $\sigma_{r r} / B$ in axial direction. 


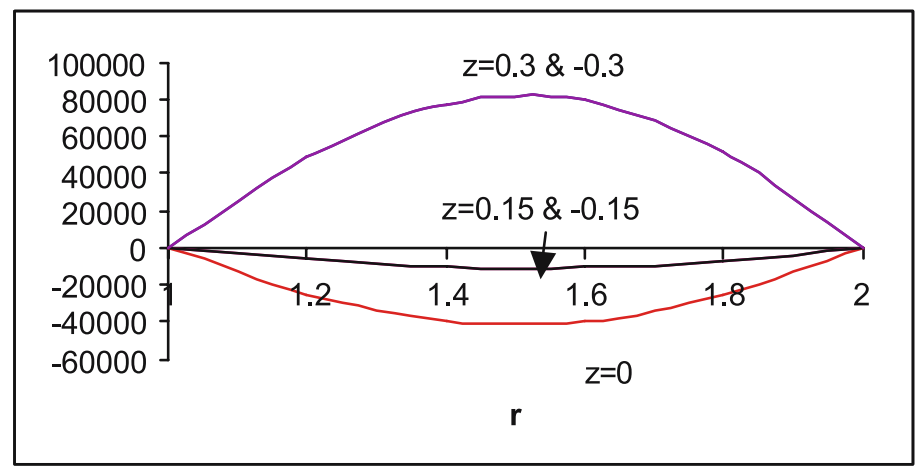

Figure 7. The stress function $\sigma_{\theta \theta} / B$ in radial direction.

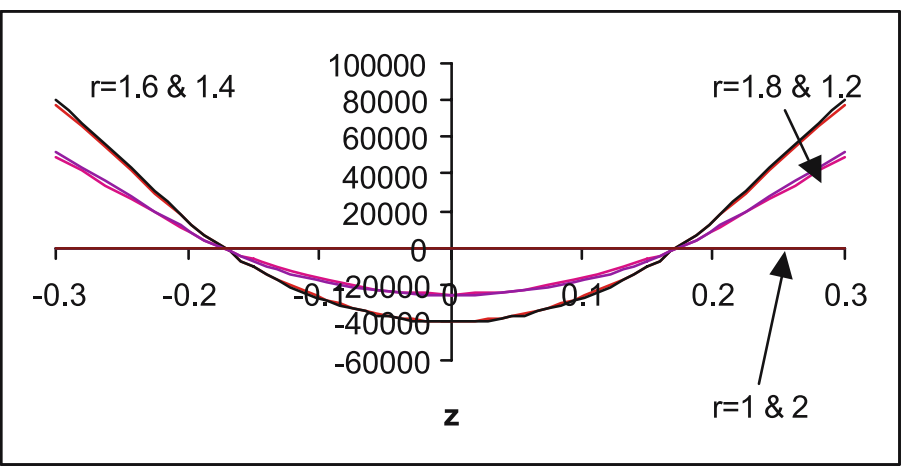

Figure 8. The stress function $\sigma_{\theta \theta} / B$ in axial direction.

\section{Conclusion}

In this paper a thick annular disc is considered which is subjected to a transient axisymmetric temperature field on the radial and axial directions of the cylindrical coordinate system and determined the expressions for temperature, displacements and stress functions, due to

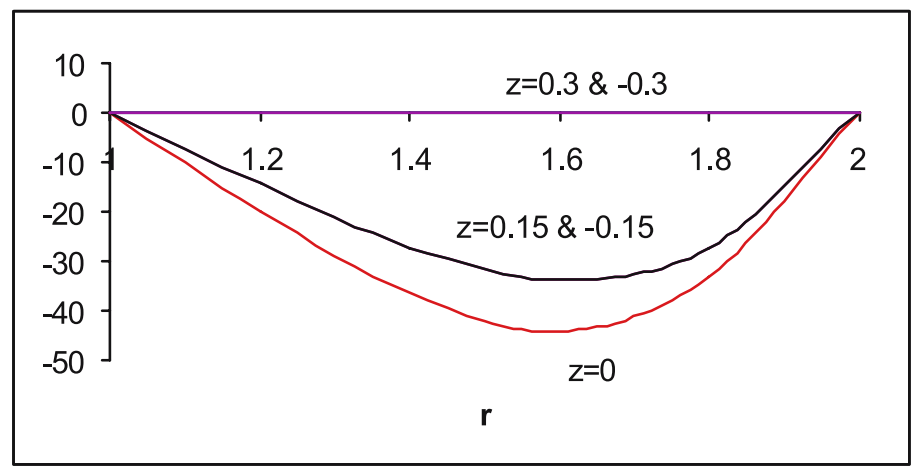

Figure 9. The axial stress function $\sigma_{z z} / B$ in radial direction. 


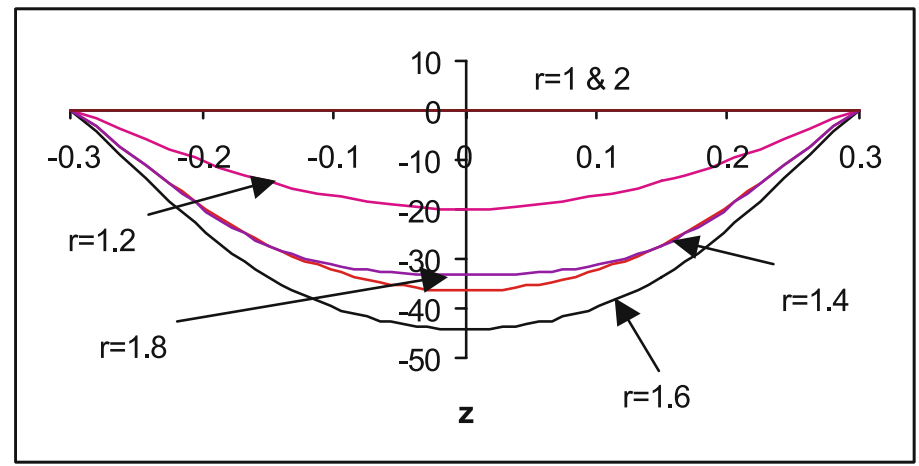

Figure 10. The axial stress function $\sigma_{z z} / B$ in axial direction.

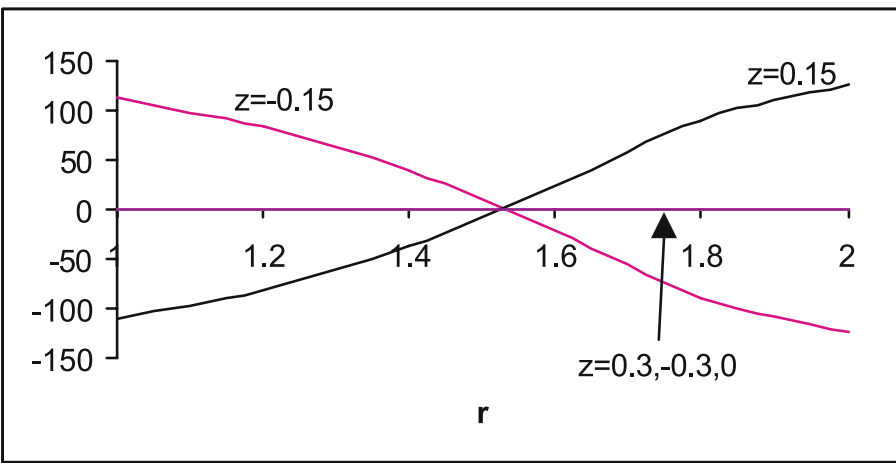

Figure 11. The stress function $\sigma_{r z} / B$ in radial direction.

arbitrary heat flux on the upper and lower surface. As a special case, mathematical model is constructed for $f(r)=\left(r^{2}-a^{2}\right)\left(r^{2}-b^{2}\right)$ and performed numerical calculations. The thermo-elastic behaviour is examined such as temperature change, displacements and stresses with the help of arbitrary heat flux on the upper and lower surface of plate.

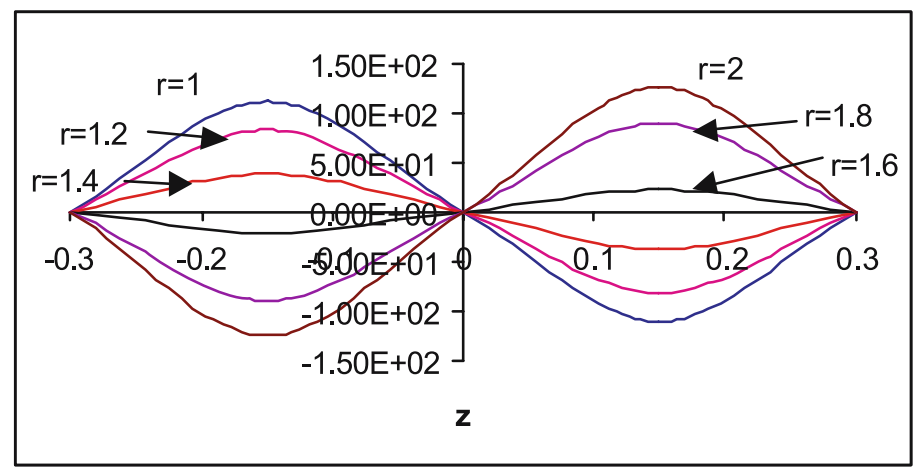

Figure 12. The stress function $\sigma_{r z} / B$ in axial direction. 
From figures 1 and 2, radial displacement decreases from inner circular surface to outer circular surface in radial direction, where as in axial direction it takes places at upper and lower surfaces of the disc.

From figures 3 and 4, axial displacement occurs at the centre i.e. $r=1.5$ in radial direction where as in axial direction decreases from lower surface to upper surface.

From figures 5 and 6, radial stress function $\sigma_{r r}$ develops tensile stress at upper and lower surface of the disc, where as it develops compressive stress in the middle of disc.

From figures 7 and 8 , the stress function $\sigma_{\theta \theta}$ develops tensile stress at upper and lower surface of the disc, where as it develops compressive stress in the middle of disc.

From figures 9 and 10, axial stress function $\sigma_{z z}$ develops compressive stress in radial as well as in axial direction. Also is zero at upper and lower surface of the disc.

From figures 11 and 12, stress function $\sigma_{r z}$ is zero at the upper, middle and lower surface of the disc. Also it is zero at center of circular region. It develops compressive stress at the upper half of the disc where as tensile stress at the lower half within $1 \leq r \leq 1.5$. Also it develops tensile stress at the upper half of the disc where as compressive stress at the lower half is within $1.5 \leq r \leq 2$.

It means we may find out that displacement and stress components occur near heat source. From the figures of radial and axial displacements it can observed that the displacements occur at the boundary surfaces of the disc. From the figures of stress functions it can observed that the compressive stress occurs at the center where as the tensile stress occurs at outer surfaces of the disc. Therefore, it may be concluded that due to arbitrary heat flux on the upper and lower surfaces of the annular disc, the disc expands towards the center $(r=0)$, and also in axial direction.

The results obtained here are useful in engineering problems particularly in the determination of state of strain in thick annular disc. Also any particular case of special interest can be derived by assigning suitable values to the parameters and function in the expression (43)-(48).

\section{References}

Ching-Huang Chiu and Cha, 0-Kuang Chen 2002 Thermal stresses in annular fins with temperature dependent conductivity under periodic boundary condition. J. Thermal Stresses 25: 475-492

Deshmukh K C 2002 Generalized transient heat conduction problem in a thin hollow cylinder. Far East J. Appl. Math. 6: 253-264

Gogulwar V S, Deshmukh K C 2002 An Inverse quasi-Static thermal stresses in an annular disc. Proc. of the ICADS (New Delhi: Narosa Publishing House)

Kulkarni V S, Deshmukh K C 2007 Quasi-static thermal stresses in a thick circular plate. J. Appl. Math. Modelling 31(8): 1479-1488

Naotake Noda, Richard B Hetnarski, Yoshinobu Tanigawa 2003 Thermal Stresses, $2^{\text {nd }}$ edn. 259-261 (New York: Taylor and Francis)

Nowacki W 1957 The state of stresses in a thick circular plate due to temperature field. Bull. Acad. Polon. Sci., Scr. Scl. Tech. 5: 227

Qian L F, Batra R C 2004 Transient thermo-elastic deformation of a thick functionally graded plate. J. Thermal Stresses 27: 705-740

Shang-Sheng Wu 1997 Analysis on transient thermal stresses in an annular fin. J. Thermal Stresses 20: 591-615

Sneddon I N 1972 The Use of Integral Transform, (New York: McGraw Hill), 235-238

Wankhede P C 1982 On the Quasi static thermal stresses in a circular plate. Indian J. Pure and Appl. Math. 13: 1273-1277 